\title{
Maraîchage et affections digestives sur le site de Houéyiho en République du Bénin
}

\author{
Elisabeth Azehoun PAZOU YEHOUENOU ${ }^{1 *}$, André SOTON ${ }^{2}$, David AZOCLI ${ }^{1}$, \\ Hortensia ACAKPO ${ }^{3}$, Hervé LAWIN ${ }^{5}$, Léonard FOURN ${ }^{4}$, Benjamin FAYOMI ${ }^{5}$, \\ Michel BOKO ${ }^{6}$, David HOUINSA ${ }^{6}$ et Jean-Claude $\mathrm{KEKE}^{7}$ \\ ${ }^{1}$ Laboratoire de Recherches en Biologie Appliquée (LARBA), Département du Génie de \\ l'Environnement, Ecole Polytechnique d'Abomey-Calavi (EPAC), Université d'Abomey-Calavi \\ (UAC), 01BP 2009 Cotonou, Bénin. \\ ${ }^{2}$ Centre Régional pour le Développement et la Santé (CREDESA) Pahou, BP 1822 Cotonou, Bénin. \\ ${ }^{3}$ Centre de Recherche d'Economie Appliqué et de Management (CREAM), Département \\ d'Economie Appliquée et de Management ENEAM-UAC, 03 BP1079 Cotonou, Bénin. \\ ${ }^{4}$ Laboratoire de Santé Communautaire FSS-UAC, BP188 Cotonou, Bénin. \\ ${ }^{5}$ Unité de Recherche et d'Enseignement en Santé au Travail et Environnement (URESTE)-FSS UAC, \\ BP188 Cotonou, Bénin. \\ ${ }^{6}$ Faculté des Lettres Art et Sciences Humaines FLASH, BP 526 Cotonou, Bénin. \\ ${ }^{7}$ Projet de Renforcement des Capacités en Conception et Analyse des Politiques de Développement \\ (CAPOD), 02 BP 534 Cotonou, Bénin. \\ *Auteur correspondant ; E-mail : yehouenoue@yahoo.fr; Tél :0022995841807
}

\section{RESUME}

Le site maraîcher de Houéyiho situé dans le domaine aéroportuaire de Cotonou connaît une importante activité maraîchère. Pour contribuer à l'amélioration de la santé des producteurs et des consommateurs et pour réduire les risques sanitaires et environnementaux, une étude a été faite en 2005. Une approche écosystémique basée sur la transdisciplinarité, la participation le genre et l'éthique a été utilisée. Des enquêtes, des observations; des discussions de groupes ont été faites auprès de 283 maraîchers (producteurs, ouvriers et vendeurs de produits maraîchers travaillant sur le site de Houéyiho). Des analyses paracliniques et celles des eaux d'arrosage ont été également faites. Les résultats de l'enquête ont montré que la situation épidémiologique des maraîchers est caractérisée par une forte fréquence des affections digestives dominées par l'amibiase (20\%) et l'anémie (36,7\%). Ces affections sont en liaison avec les germes pathogènes tels que : Entamoeba histolitica, Ankylostoma duedonalea contenus dans les eaux utilisées pour arroser les planches. Pour faire face aux pathologies identifiées, les maraîchers ont été traités, des latrines sont construites sur le site et des sensibilisations sur l'hygiène ont été faites avec des résultats encourageants.

(c) 2013 International Formulae Group. All rights reserved.

Mots clés : Maraîchage, affections digestives, Houéyiho, Cotonoou, Bénin.

\section{INTRODUCTION}

Le maraîchage est une activité informelle génératrice de revenu et d'emploi pratiquée par les couches vulnérables de régions urbaines et périurbaines (Lachance, 1999). Selon les estimations de l'Organisation 
des Nations Unies pour l'Alimentation et l'Agriculture (FAO), environ 800 millions de personnes dépendent de l'agriculture urbaine (Marc Chevrier, 2001).

$\mathrm{Au}$ Bénin et plus particulièrement à Cotonou, on dénombre plusieurs sites maraîchers dont le plus important est celui de Houéyiho qui a une superficie de 15 hectares. La couche de la population qui s'adonne à cette agriculture urbaine est constituée d'une diversité de groupes socioculturels, formés de paysans immigrés ruraux, d'ouvriers, d'apprentis, de commerçants et d'aides familiales. Créé en 1972, ce site ne comptait que 34 exploitants. En 2005 ils étaient au nombre de 283 dont 222 hommes et 61 femmes répartis au sein de 5 groupements (Tableau 1). Il résulte de cette situation une occupation anarchique des marais, des dépressions et des espaces vides. Les sols très sableux et pauvres en matière organique exigent des apports élevés en intrants (Pazou Yehouenou et al., 2010). Les maraîchers utilisent des ordures ménagères non traitées, des fientes de volaille, des matières fécales provenant des porcs élevés sur le site et des pesticides inappropriés surtout ceux destinés au traitement de coton. Tous ces facteurs ne garantissent pas la qualité des légumes produits (Assogba-Komlan et al., 2007). Selon Aubry et al. (2010), la proximité urbaine est susceptible d'engendrer des risques spécifiques sur les produits de l'agriculture urbaine. Ainsi, les conditions peu hygiéniques dans lesquelles sont cultivées les produits maraîchers surtout les légumes feuilles, et les nombreuses maladies digestives enregistrées constituent un problème de santé publique aussi bien au Bénin que dans les villes africaines comme à Dakar et à Yaoundé (Kenmogne et al., 2010 ; Gaye et al., 2002).

$\mathrm{Au}$ Bénin, certaines infections digestives sont attribuées à tort ou à raison à la consommation des produits maraîchers ce qui entraîne des méfiances vis-à-vis de ceux-ci et parfois des méventes. Eu égard à tout ce qui précède et pour améliorer la santé des consommateurs, des producteurs, une équipe de chercheurs (hommes et femmes), ayant différentes spécialités, associée aux maraîchers et maraîchères a utilisé une approche écosystémique basée sur la transdisciplinarité, la participation, le genre et l'éthique. L'objectif général est d'améliorer l'état de santé des maraîchers et des consommateurs et de façon spécifique :

- déterminer au niveau des maraîchers la fréquence des atteintes digestives,

- faire le lien entre le niveau de contamination des eaux d'arrosage des produits maraîchers et l'état de santé des producteurs.

\section{MATERIEL ET METHODES Milieu d'étude}

Le site maraîcher de Houéyiho est situé sur le cordon littoral (MEHU, 1993) avec des sols à gley salés, hydromorphes peu perméables et peu fertiles car ils sont dépourvus d'humus. Le périmètre de Houéyiho est sous l'influence du climat subéquatorial caractérisé par l'alternance de deux saisons pluvieuses (avril à juillet et de septembre à octobre) et deux saisons sèches (fin juillet à août et novembre à mars). Les précipitations à Cotonou sont en général constituées d'averses orageuses et de pluies diluviennes pouvant durer parfois plus de vingt-quatre heures. Les hauteurs de pluie enregistrées à Cotonou pendant la grande saison pluvieuse varient entre 300 et $1000 \mathrm{~mm}$ selon les années.

\section{Echantillonnage}

Un recensement des hommes, des femmes et des ouvriers travaillant sur le site est fait groupement par groupement afin de constituer une base de données. Ceux qui ont une expérience de moins de deux ans ne sont pas retenus pour prendre part aux examens cliniques et paracliniques. L'échantillonnage des hommes est fait de manière systématique avec un pas de 1 sur 2. Toutes les femmes sont retenues à l'exception de celles qui ont une expérience inférieure à deux ans dans le domaine. Seules les commerçantes qui fréquentent de façon permanente le site durant la période d'enquête sont toutes retenues. 


\section{Enquêtes}

L'administration de questionnaire, des entretiens de groupe auprès des marâichers, des commerçants et des ouvriers se sont déroulés en langues nationales pour éviter les biais d'interprétation. Des observations directes sont faites sur le site. Les enquêtes cliniques ont consisté à rechercher les cas d'intoxication due aux maladies infectieuses ou parasitaires ou due aux produits toxiques.

\section{Examens paracliniques individuels}

Les examens paracliniques effectués sont l'examen des selles fraîches et la coproculture. L'examen direct des selles est effectué et est complété par la technique de Kato Katz.

\section{Etude de l'hémogramme}

Il a été réalisé sur l'automate SYSMEX 9000 et a permis de déterminer les composantes hématologiques.

\section{Analyses bactériologiques parasitologiques des eaux d'arrosage}

et

Elles sont faites sur les eaux d'arrosage prélevées dans les marécages, les puits et les forages.

\section{Considération d'ordre éthique}

Une autorisation du comité national d'éthique a été obtenue avant les prélèvements. Les maraîchers ont été informés sur les types de prélèvements à faire. Le consentement individuel écrit et signé a été obtenu auprès de chaque maraîcher. Il leur a été garanti une confidentialité des résultats des analyses.

\section{Analyses statistiques}

Les données sont saisies avec les moyens informatiques et traitées à l'aide des logiciels SPSS/PC + et SPAD version 4.01. Des comparaisons ont été faites à l'aide du test de Khi-deux. On a procédé également à l'analyse multivariée.

Tous les examens sont repris tous les 6 mois sur une période de deux ans.

\section{La validation et la restitution}

Plusieurs réunions de validation ont porté sur la présentation des résultats des travaux des sous équipes et sur la validation des rapports. Les observations des uns et des autres sont prises en compte. Plusieurs séances de restitution des analyses cliniques et paracliniques et les résultats des analyses de l'eau sont effectuées. Toutes les affections parasitaires et infectieuses diagnostiquées sont traitées.

Tableau 1 : Répartition des maraîchers par sexe, par groupements et par zone.

\begin{tabular}{lcccc}
\hline ZONE & Groupements & \multicolumn{3}{c}{ EFFECTIFS } \\
\cline { 3 - 5 } & & Hommes & Femmes & TOTAL \\
\hline \multirow{2}{*}{ NORD } & Grâce de Dieu & 28 & 12 & 40 \\
& Enagnon & 59 & 13 & 72 \\
& Kponhami & 36 & 6 & 42 \\
\hline SUD & Semèvo & 61 & 13 & 74 \\
& Wangnina & 38 & 17 & 55 \\
\hline TOTAL & 222 & 61 & 283 \\
\hline \multicolumn{2}{l}{ Le Tableau 1 montre que 283 maraîchers travaillent sur le site avec une répartition de 222 hommes pour 61 femmes } \\
au niveau des 5 groupements qui existent.
\end{tabular}




\section{RESULTATS}

Résultats des enquêtes

Les interventions menées sur le site après les résultats des analyses cliniques et paracliniques

Les résultats des analyses effectuées sont remis aux personnes sélectionnées pour l'enquête; certaines affections aiguës (anémie, parasitose, etc.) sont traitées avec distribution de médicaments. En ce qui concerne les personnes chez qui il a été décelé des affections chroniques (hypertension, dermatose, hernie, etc.), elles ont été orientées vers les centres de santé de référence de leur localité de résidence pour une prise en charge.

L'équipe de recherche et les maraîchers ont construit sur le site 7 latrines et urinoirs sur fonds propres et avec l'aide technique des partenaires au développement comme le CREPA, la GIZ.

Tous les maraîchers du site ont été sensibilisés sur les règles d'hygiène à observer (lavage des mains et des plats avant de manger, ne pas déféquer dans les basfonds et à l'air libre).

La Figure 1 illustre l'état de santé des maraîchers et des ouvriers entre le premier et le second examen. En effet, toutes les affections mises en évidence lors du deuxième examen ont connu une régression spectaculaire de leur fréquence.
La Figure 2 illustre les différents symptômes et affections enregistrés au troisième passage. La fréquence de la diarrhée aiguë a régressé. Celle de la dysenterie a suivi la même tendance que la diarrhée mais avec une fréquence presque identique aux deux premiers passages.

Entre le troisième et le quatrième passage (Figure 3), la fréquence des diarrhées et des dysenteries a diminué. Par contre, les infections respiratoires exprimées par la toux ont légèrement augmenté de fréquence. Il en est de même des douleurs lombaires. La fréquence des cas d'hypertension artérielle n'a pas beaucoup évolué mais les intéressés sont sous traitement. Les autres symptômes ont une fréquence moindre au quatrième passage. Au total, il y a moins de marâichers malades au quatrième passage qu'au troisième comme le montre la Figure 3.

D'une façon globale on constate une concentration relativement élevée des cas de malades porteurs d'Entamoeba histolytica. L'examen bactériologique de l'eau d'arrosage indique une forte fréquence d'Entamoeba histolytica. Le rapprochement de ces données de celles de l'analyse bactériologique de l'eau d'arrosage des légumes sur le site laisse supposer une contamination par des matières fécales. La consommation des légumes arrosés avec cette eau peut bien entraîner des troubles digestifs.

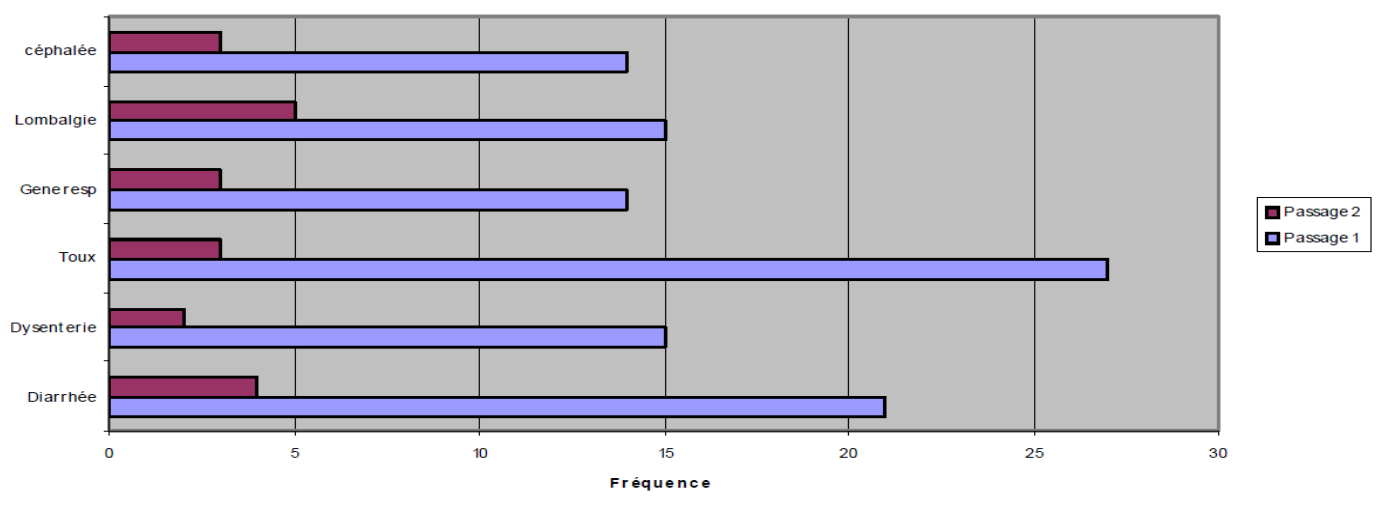

Figure 1 : Comparaison de la distribution des affections enregistrées entre le premier et le deuxième passage. 


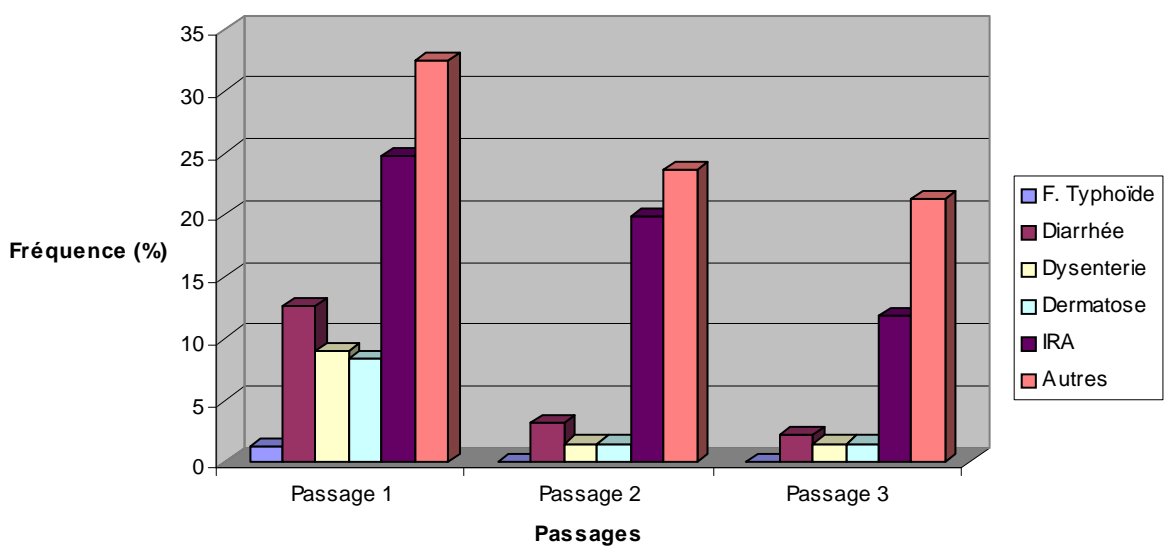

Figure 2 : tendance évolutive des troubles enregistrés aux trois passages.

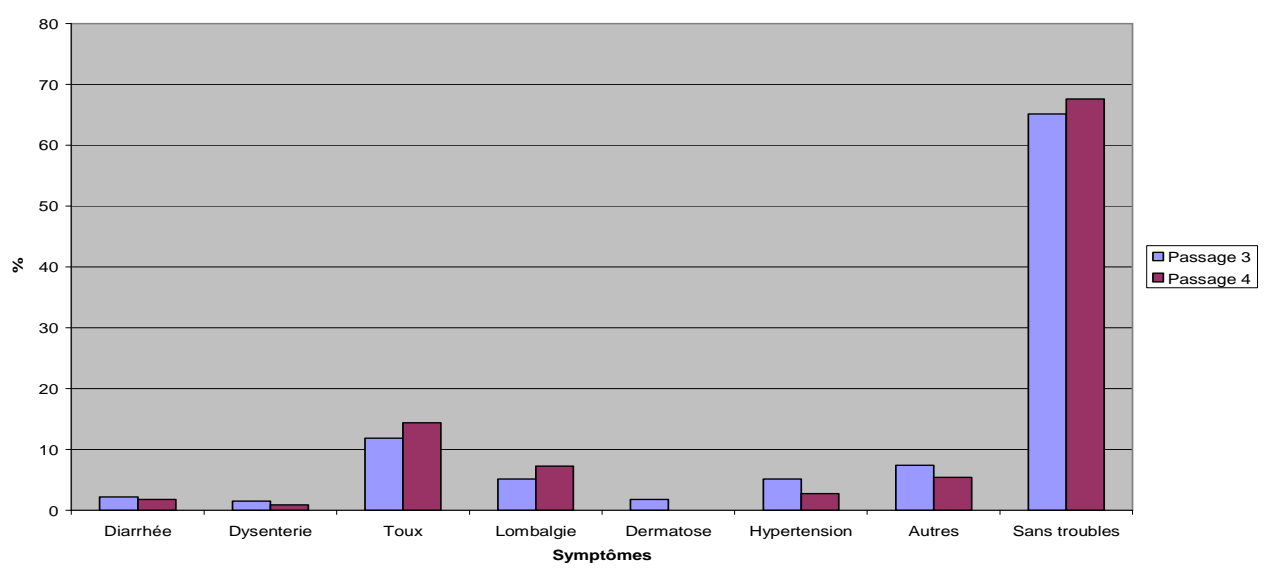

Figure 3 : Evolution des symptômes entre les passages 3 et 4 .

\section{DISCUSSION}

\section{Troubles digestives}

La diarrhée est plus enregistrée chez les maraîchers $(12,7 \%)$ que chez les ouvriers ; il en est de même des cas de dysenteries avec des selles striées de sang (Tableau 2). Ces fréquences élevées sont probablement liées au manque d'hygiène alimentaire des exploitants par exemple l'habitude de ne pas se laver les mains avant de manger, les bols et plats qui sont rincés avec les eaux des puits à ciel ouvert. Parmi les autres signes et symptômes enregistrés, l'interrogatoire des maraîchers a permis de constater que $4,8 \%$
( $n=166)$ d'entre eux ont été hospitalisés pour fièvre traînante au cours des trois derniers mois précédant l'enquête. $9 \%$ des maraîchers souffrent de lombalgies et $4,2 \%$ de douleurs musculo-squelettiques. De plus, les cas d'irritation ou d'hyperhémie conjonctivale $(9,6 \%)$, de polynévrite et d'hypertension artérielle et autres $(8,4 \%)$ ont été enregistrés chez ces mêmes maraîchers. Par ailleurs, 6,7\% des commerçantes ont déclaré avoir eu une fièvre traînante sans autres signes d'accompagnement. Une commerçante sur dix souffre de douleurs musculo-squelettiques liées probablement au déplacement 
intempestif pour l'approvisionnement des légumes sur le site et surtout pour la vente des produits maraîchers en ville. Les amibiases identifiées chez les maraîchers de Houéyiho $(20,7 \%)$ sont nettement inférieurs à ceux observés chez les maraîchers de Ouakam (42,5\%), de Pikine (35\%) au Sénégal et de Abiergué à Yaoundé au Cameroun (61,5\%) (Kenmogne et al., 2010). Les résultats des examens cliniques et paracliniques des maraîchers de Houéyiho, comparés à ceux de Ouakam et Pikine au Sénégal, montrent par exemple que le taux d'anémie observé chez les maraîchers de Houéyiho (18,2\%) est inférieur à celui des marâichers de Ouakam qui est de $61 \%$.

\section{Troubles parasitaires}

Le Tableau 3 présente les différents troubles parasitaires observés aussi bien chez les maraîchers que chez les ouvriers. L'examen des selles par la technique de Kato Katz indique une fréquence relativement élevée de l'amibiase intestinale chez les maraîchers $(20,7 \%)$ et chez les ouvriers $(23,7 \%)$. L'ankylostomiase vient en seconde position et concerne dans des proportions presque similaires, les mêmes groupes de maraîchers (7 à 8\%).

Sur le site, il est aisé de constater que rares sont les maraîchers et les ouvriers qui portent des chaussures lors de l'arrosage ou du semis de leurs planches enrichis d'engrais de toutes sortes. Les ankylostomes, profitant de la marche pieds nus, pénètrent l'organisme des maraîchers par voie transcutanée et provoquent des troubles. L'anémie est l'expression clinique de cette infection parasitaire du fait qu'un ankylostome se nourrit par jour de $0,02 \mathrm{ml}$ de sang qu'il suce au niveau de l'intestin du sujet parasité. Les ascaris sont peu représentés soit isolément ou en association. Par contre, le polyparasitisme est plus remarqué chez les maraîchers avec une prédominance du couple amibe ankylostome (2,4\%). Par ailleurs, les commerçantes sont peu parasitées comme l'illustre le Tableau 3 Cela peut supposer qu'elles se déparasitent régulièrement ou sont moins exposés au manque d'hygiène alimentaire que connaissent les maraîchers sur le site.

De façon générale, les prévalences des parasites intestinaux tels que les trichocéphales, les Giardia intestinalis, l'ascaris et les amibes etc se retrouvent aussi bien chez les maraîchers du Sénégal que ceux du Bénin avec 37,7\% à Houéyiho, $60 \%$ à Ouakam et $41,2 \%$ à Pikine.

Ces troubles digestifs ont été également relevés par des auteurs dans d'autres villes des pays en développement (Nguegang, 2008 ; Cissé et al., 2002 ; Kengne et al., 2002 ; Sonou, 2001).

\section{Troubles hématologiques}

Ces troubles anémiques peuvent expliquer la fatigue intense déclarée par certains des maraîchers et ouvriers. Les ouvriers présentent également un fort taux de neutropénie $(25,8 \%)$ alors que seulement 19,3\% des maraîchers en sont concernés. Ces mêmes taux s'observent au niveau des mêmes exploitants pour la thrombopénie. Par ailleurs, l'hyper-éosinophilie, indicateur d'une parasitose intestinale probable, est présente dans le sang aussi bien des maraîchers $(6,6 \%)$ que des ouvriers $(9,7 \%)$ en des proportions variables. Quant à la prévalence de lymphopénie, elle est globalement faible (Tableau 4) Les données de l'examen du sang chez les exploitants prélevés sur le site sont enregistrées dans le Tableau 4 et indiquent que plus d'un maraîcher sur dix $(10,8 \%)$ souffre d'une anémie légère (taux d'hémoglobine inférieur à $12 \mathrm{~g} / \mathrm{L})$ à prédominance ferriprive. Les ouvriers $(12,9 \%)$ et les commerçantes (30\%) apparaissent plus atteints que les maraîchers.

En considérant uniquement le groupe des femmes, on constate que la plupart d'entre elles sont anémiées. Parmi elles, on remarque que les plus nombreuses sont les maraîchères (20\%) avec un taux d'hémoglobine inférieur à 


\section{E. A. PAZOU YEHOUENOU et al. / Int. J. Biol. Chem. Sci. 7(5):1976-1986, 2013}

$12 \mathrm{~g} / \mathrm{l}$. Celles qui présentent une neutropénie ou une thrombopénie sont dans des proportions égales à $6 \%$. Pour la lymphopénie, les porteuses sont peu nombreuses $(3 \%)$.

\section{Modèle de régression logistique}

Le Tableau 5 montre le modèle de régression logistique et a permis de mettre en évidence la contribution des caractéristiques individuelles des maraîchers et le système de production sur le site pour la compréhension de la fréquence des troubles digestifs enregistrés.

Les résultats de cette analyse multivariée indiquent que les hommes sont plus atteints que les femmes. Mais à âge égal, le risque d'avoir une infection digestive est presque le même. L'état matrimonial intervient très faiblement pour expliquer l'apparition des troubles digestifs chez les maraîchers. Par contre la durée de séjour sur le site est inversement liée au risque des maladies digestives. En effet, plus la durée de séjour est longue moins le risque de contamination digestive apparaît grand. Cependant, le statut des exploitants (maraîchers, ouvriers) induit deux fois plus le risque de troubles digestifs sur le site.

En ce qui concerne les utilisateurs de pesticides, ils sont également exposés au risque de diarrhée ou de dysenterie; ils courent plus de risque que ceux qui n'en utilisent pas. Ainsi, l'association de l'apparition de la maladie et la «survie des maraîchers » indique que les maraîchers exposés courent une fois et demi plus de risque que les autres $(\mathrm{OR}=1,40)$. La nature du matériel d'arrosage intervient très peu dans l'apparition des infections digestives chez les maraîchers. Quant au temps d'arrosage, plus il est long, plus le maraîcher court un risque d'infection digestive (diarrhée ou dysenterie). Néanmoins, l'expérience professionnelle peut permettre de réduire ce risque. Le type de légumes produits et consommés ne semble pas constituer un facteur de risque de la maladie
$(\mathrm{OR}<1)$. Cependant, les fertilisants utilisés par les maraîchers apparaissent comme facteur de risque de contamination dont la capacité discriminante est environ deux fois plus importante chez les malades que chez les non malades $(\mathrm{OR}=2,55)$.

La corrélation entre la mauvaise qualité des eaux et la recrudescence de l'amibiase intestinale, en particulier les maladies hydriques qui affectent les maraîchers y trouve des éléments de réponse. Cette recrudescence de maladies hydriques a été étudiée par plusieurs auteurs dans différentes villes des pays en développement : Rutkowski et al. (2007) au Katmandu, Van Der Hoek et al. (2005) à Phnom Penh. Il va sans dire que les maladies hydriques dans cette zone découlent également de la réutilisation des eaux usées dans le maraîchage. Ces risques sanitaires sont malheureusement exacerbés par de nombreux comportements et pratiques à risques, l'absence d'équipements appropriés et le contact permanent avec les eaux usées.

\section{Comparaison des troubles, des affections et des symptômes enregistrés après intervention}

Les Figures 1, 2 et 3 montrent une diminution des troubles et affections préalablement enregistrées sur le site. La diarrhée a été la plus sensible aux mesures de préventions suggérées. La fréquence de la dysenterie a présenté une allure similaire à la diarrhée, mais sa diminution paraît lente. Les déclarations des ouvriers lors de leur examen clinique a montré un seul cas de dysenterie avec du sang dans les selles depuis le premier passage. Aucun cas de diarrhée n'est rapporté chez les ouvriers. En dehors de ces deux affections digestives liées à la consommation d'eau ou d'aliments contaminés par les matières fécales, les maraîchers et les ouvriers ont reconnu une diminution de leurs douleurs lombalgiques. L'intensité de la douleur lombalgique n'a pas entravé leurs activités habituelles sur le site. D'autres symptômes ont été signalé tels les céphalées et les courbatures 
d'origine palustre (le site étant dans une zone d'endémicité palustre) puis le vertige d'origine peu élucidée. Les troubles respiratoires exprimés par la toux après pulvérisation de pesticide ne sont signalés par aucun de ces ouvriers examinés. Les cas de fièvre typhoïde enregistrés et traités au premier passage sont guéris sans récidive. Il n'y a plus d'autres cas enregistrés aux autres passages. Pour ce qui concerne les infections respiratoires aiguës (IRA) et les autres troubles, leur fréquence moindre par rapport au premier tour demeure néanmoins élevée à chaque passage.

\section{Comparaison des affections parasitaires}

Le Tableau 6 montre les cas de parasitoses enregistrés au cours des quatre analyses effectuées. Au premier passage cinq espèces de parasitoses intestinales étaient identifiées chez les maraîchers avec une dominance des Entamoeba (20,7\%) suivi des ankylostomes $(7,3 \%)$ et des associations parasitaires (polyparasitisme).

$\mathrm{Au}$ second passage, seuls trois des cinq espèces ont été retrouvées avec une fréquence réduite de près de la moitié. En comparant ces résultats à ceux rapportés au démarrage de l'étude, on constate une diminution de $73,5 \%$ des infections amibiennes et de $75 \%$ pour l'ankylostomiase. Quant aux anguillules, le taux de variation est de $100 \%$ alors que le trichocéphale a diminué seulement de moitié.

$\mathrm{Au}$ troisième passage les amibes ont persisté avec une fréquence presque identique qu'au second passage mais largement moindre par rapport au premier passage. Ces résultats montrent que les actions sanitaires menées (traitement des cas, conseils d'hygiène des mains et des selles puis des aliments) sur le site de Houéyiho ont réussi à réduire la fréquence des infections. En comparant ces résultats à ceux rapportés aux différents passages antérieurs (Tableau 6), on constate une réapparition des œufs d'ascaris absents aux seconds et troisièmes examens de selles. L'ankylostomiase réapparut avec $2 \%$ de cas contre 0 cas au troisième passage et $3,3 \%$ au second passage, soit une diminution de fréquence relative d'environ un point. Le risque d'amibiase est toujours présent chez les maraîchers, mais avec une faible intensité par rapport au passage précédent. En effet, de $11 \%$ au troisième passage, la proportion de maraîchers parasités aux «Entamoeba » a baissé d'environ $6 \%$.

Tableau 2: Symptômes enregistrés au niveau des maraîchers, ouvriers et commerçants de Houéyiho.

\begin{tabular}{lccc}
\hline Affections & $\begin{array}{c}\text { Maraîchers } \\
(\mathbf{n = 1 6 6})\end{array}$ & $\begin{array}{c}\text { Ouvriers } \\
(\mathbf{n = 3 1})\end{array}$ & $\begin{array}{c}\text { Commerçantes } \\
(\mathbf{n = 3 0})\end{array}$ \\
\hline Fièvre traînante & 8 & 2 & 2 \\
Diarrhée & 21 & 3 & 0 \\
Dysenteries (sang dans les selles) & 15 & 0 & 0 \\
Toux & 27 & 8 & 1 \\
Gène respiratoire & 14 & 3 & 1 \\
Eczéma & 14 & 1 & 0 \\
Douleur Musculo-squelettique & 7 & 0 & 3 \\
Polynévrite & 2 & 0 & 0 \\
Lombalgie & 15 & 1 & 1 \\
Irritation/hyperhémie oculaire & 16 & 0 & 0 \\
Autres (Hypertension artérielle, hernie, asthme.) & 14 & 1 & 1 \\
\hline
\end{tabular}

Les résultats des signes et symptômes enregistrés lors du premier passage sont consignés dans le Tableau 2. 
Tableau 3: Fréquence des espèces de parasites selon le type d'exploitants.

\begin{tabular}{lccc}
\hline Parasites & $\begin{array}{c}\text { Maraîchers } \\
(\mathbf{n = 1 6 6})\end{array}$ & $\begin{array}{c}\text { Ouvriers } \\
(\mathbf{n}=\mathbf{3 0})\end{array}$ & $\begin{array}{c}\text { Commerçantes } \\
(\mathbf{n}=\mathbf{3 0})\end{array}$ \\
\hline Ascaris & 1 & 1 & 0 \\
Ankylostome & 12 & 5 & 0 \\
Anguillule & 2 & 0 & 0 \\
Amibes (Entamoeba) & 34 & 14 & 2 \\
Amibes +Ascaris & 1 & 0 & 0 \\
Amibes +Ankylostome & 4 & 2 & 0 \\
Amibe + trichocéphale & 2 & 1 & 0 \\
Eufs de bilharziose & 0 & 2 & 0 \\
\hline
\end{tabular}

Les différentes espèces de parasites enregistrées selon le type d'exploitants sont consignées dans le Tableau 3.

Tableau 4 : Résultats hématologiques.

\begin{tabular}{lccccc}
\hline Paramètres & $\begin{array}{c}\text { Maraîchers } \\
(\mathbf{n = 1 1 2})\end{array}$ & $\begin{array}{c}\text { Maraîchères } \\
(\mathbf{n = 5 8})\end{array}$ & $\begin{array}{c}\text { Ouvriers } \\
(\mathbf{n = 2 6})\end{array}$ & $\begin{array}{c}\text { Ouvrières } \\
(\mathbf{n = 4})\end{array}$ & $\begin{array}{c}\text { Commerçantes } \\
(\mathbf{n = 3 0})\end{array}$ \\
\hline Anémie $(\mathrm{Hb}<12 \mathrm{~g} / \mathrm{l})$ & 18 & 13 & 4 & 1 & 9 \\
Neutropénie & 32 & 4 & 8 & & 1 \\
Thrombopénie & 27 & 4 & 3 & 2 & 1 \\
$\begin{array}{l}\text { Hyperoésinophilie } \\
\text { Lymphopénie }\end{array}$ & 11 & & 3 & & 1 \\
$\begin{array}{l}\text { Autres (polyglobulie, } \\
\text { polynucléose) }\end{array}$ & 1 & 2 & 1 & & 2 \\
Normaux & 73 & 42 & 0 & & 1 \\
\multicolumn{1}{c}{ Les différents troubles hématologiques observés selon le type d'exploitants sont consignés dans le Tableau 4.}
\end{tabular}

Tableau 5 : Modèle de régression pour l'analyse de la relation entre maladies digestives et caractéristiques des individus et de la production.

\begin{tabular}{lccc}
\hline Caractéristiques & Bêta & Erreur type & Odds Ratio \\
\hline Sexe & $-0,59$ & 0,66 & 0,55 \\
Age & 0,03 & 0,02 & 1,03 \\
Etat matrimonial & 0,11 & 0,26 & 1,11 \\
Durée séjour & $-0,21$ & 0,29 & 0,81 \\
Diplôme & $-0,08$ & 0,22 & 0,92 \\
Statut (maraîchers/ouvriers) & 0,81 & 0,60 & 2,25 \\
Utilisation de pesticide & 0,17 & 0,12 & 1,18 \\
Survie (non apparition de & 0,33 & 0,70 & 1,40 \\
maladies) & & & \\
Matériel d'arrosage & 0,09 & 0,35 & 1,09 \\
Durée de l'arrosage & $-0,03$ & 0,21 & 0,97 \\
Technique d'arrosage & $-1,70$ & 1,27 & 0,18 \\
Types de légumes & $-0,44$ & 0,08 & 0,96 \\
Fertilisants & 0,93 & 0,57 & 2,55 \\
\hline
\end{tabular}


Tableau 6 : Evolution des infections parasitaires des maraîchers.

\begin{tabular}{lcccc}
\hline $\begin{array}{l}\text { Passage } \\
\text { Parasites }\end{array}$ & $\begin{array}{c}\text { Passage 1 } \\
\text { N=166 }\end{array}$ & $\begin{array}{c}\text { Passage 2 } \\
\mathbf{N = 9 2}\end{array}$ & $\begin{array}{c}\text { Passage 3 } \\
\mathbf{N = 1 3 6}\end{array}$ & $\begin{array}{c}\text { Passage 4 } \\
\mathbf{N = 1 0 0}\end{array}$ \\
\hline Ascaris & 1 & 0 & 0 & 2 \\
\hline Ankylostomes & 12 & 3 & 0 & 2 \\
\hline $\begin{array}{l}\text { Amibes } \\
(\text { Entamoeba })\end{array}$ & 34 & 9 & 15 & 6 \\
\hline Trichocéphale & 2 & 1 & 0 & 0 \\
\hline Giardia & 0 & 0 & 0 & 2 \\
\hline Douve & 0 & 2 & 0 & 0 \\
\hline Anguillule & 2 & 2 & 0 & 0 \\
\hline Le Tableau 6 montre l'évolution des infections parasitaires chez les maraîchers aux 4 passages.
\end{tabular}

Par ailleurs, absentes des autres examens de selles effectués jusqu'au quatrième passage, des formes végétatives de giardia sont enregistrées. La comparaison de ces résultats généralement à la baisse souligne sans aucun doute, l'effet des interventions préventives sur le terrain à savoir le déparasitage de sujets parasités après les résultats d'examen de selles et les conseils d'hygiène alimentaire à travers les séances d'éducation pour la santé.

$\mathrm{Au} 4^{\text {ème }}$ passage, on note une prédominance d'Entamoeba histolitica (6\%), responsable des dysenteries amibiennes. Quant à l'ankylostomiase, cause d'anémie généralement ferriprive, elle est retrouvée chez $2 \%$ avec une faible quantité d'œufs allant de 8 à 14 à l'examen de Kato. Deux pour cent (2\%) d'œufs d'ascaris ont été observés avec une quantité moindre d'œufs. Aussi des formes végétatives de Giardia (2\%) sont mises en évidence. Par ailleurs, quelques cas d'Entamoeba non pathogènes sont retrouvés dans $13 \%$. Enfin, aucun cas de trichocéphalose n'est identifié chez ces acteurs du site de Houéyiho.

\section{REMERCIEMENTS}

L'équipe de recherche témoigne sa gratitude au CRDI qui lui a permis de réaliser cette étude grâce à un soutien financier. Nos remerciements vont également aux partenaires comme la GIZ et l'EAA (ex CREPA) ainsi qu'aux maraîchers qui sont désormais nos collaborateurs.

\section{REFERENCES}

Assogba-Komlan F, Ahlé V, Azagba J. 2002. Apport raisonné des engrais organiques pour une bonne productivité des légumes feuilles en sols sableux marins. In Actes de l'Atelier Scientifique 3. Programme Régional Sud-Centre du Bénin : Bénin ; 150-157.

Aubry C, Dabat M-H, Mawois M. 2010. Fonction alimentaire de l'agriculture urbaine au Nord et au Sud: Permanence et renouvellement des questions de recherche. Rapport ISDA, Montpellier, 230 Juin 2010.

Cissé G, Kientga M, Ouedraogo B, Tanner M. 2002. Développement du maraîchage autour des eaux de barrage à Ouagadougou: quels sont les risques sanitaires à prendre en compte? Cahiers d'Etudes et de Recherches Francophones. Agricultures, 11(1): 31-38.

Kenmogne K, Romain G, Rosillon F, Mpakam HG, Nono A. 2010. «Enjeux sanitaires, socio-économiques et environnementaux liés à la réutilisation des eaux usées dans le maraîchage urbain: cas du bassin versant de l'Abiergué (Yaoundé-Cameroun)», VertigO - La Revue Electronique en 
Sciences de l'Environnement, 10(2): http://vertigo.revues.org/10323.

Kengne IM, Endamana D, Soh L, Nya J. 2002. Réutilisation des eaux usées pour l'agriculture urbaine à Yaoundé. Actes de l'atelier international CTA/ETC RVAF/CREPA sur la réutilisation des eaux usées en agriculture urbaine. Ouagadougou, 116-123.

Lachance A. 1999. Cultiver en ville. In Ressources Explore (vol 21, N³). CRDI : Pays-Bas.

Marc Chevrier. 2001. L'agriculture urbaine ou les paysages nourriciers de la ville. Agora, 8(3) : 37-39.

MEHU (Ministère de l'Environnement de l'Habitat et de l'Urbanisme). 1993. Plan d'action environnemental du Bénin, rapport d'activités, MEHU, $134 \mathrm{p}$.

Nguegang AP. 2008. L'agriculture urbaine et péri-urbaine à Yaoundé : analyse multifonctionnelle d'une activité montante en économie de survie. Thèse de Doctorat, Université Libre de Bruxelles, $200 \mathrm{p}$.
Pazou Yehouenou EA, Soton A, Azocli D, Acakpo H, Boco M, Fourn L, Houinsa D, Keke J-C, Fayomi B. 2010. Contamination du sol, de l'eau et des produits maraîchers par des substances toxiques et des métaux lourds sur le site de Houéyiho (Cotonou) en République du Bénin. Int. J. Biol. Chem. Sci., 4(6) : 2160-2168.

Rutkowski T, Raschid-Sally L, Buechler S, 2007. Wastewater irrigation in the developing world Two case studies from Katmandu Valley in Nepal. Agricultural Water Management, 88: 83-91.

Sonou M. 2001. Periurban agriculture and health risks in Ghana. UAM., 1(3): 33-34.

Van Der Hoek W, Tuan Anh V, Cam PD, Vicheth C, Dalsgaard A. 2005. Skin diseases among people using urban wastewater in Phnom Penh. Urban Agriculture Magazine, 14: 30-31. 